\title{
Combined use of hard X-ray phase contrast imaging and X-ray fluorescence microscopy for sub-cellular metal quantification
}

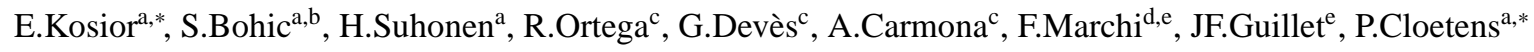 \\ ${ }^{a}$ European Synchrotron Radiation Facility, Grenoble, France \\ ${ }^{b}$ INSERM U-836 (Team 6:Synchrotron Radiation and Medical Research), Grenoble Institut of Neuroscience, Grenoble, France \\ ${ }^{c}$ Centre d'Etudes Nucléaires de Bordeaux Gradignan, CENBG, CNRS- Université de Bordeaux 1, Gradignan, France \\ ${ }^{d}$ Physics Department, UFR PHITEM, University of Joseph Fourier, Grenoble, France \\ ${ }^{e}$ Surface Science Laboratory at the European Synchrotron Radiation Facility, Grenoble, France
}

\begin{abstract}
Hard X-ray fluorescence microscopy and magnified phase contrast imaging are combined to obtain quantitative maps of metal concentration in whole cells. The experiments were performed on freeze dried cells at the nano-imaging station ID22NI of the European Synchrotron Radiation Facility (ESRF). X-ray fluorescence analysis gives the areal mass of most major, minor and trace elements; it is validated using a biological standard of known composition. Quantitative phase contrast imaging provides maps of the projected mass. the phase retrieval step is validated using calibration samples and through comparison with non-X-ray techniques (Atomic Force Microscopy, Scanning Transmission Ion Microscopy). Up to now, absolute quantification at the sub-cellular level was impossible using X-ray fluorescence microscopy but can be reached with the use of the proposed unique approach.
\end{abstract}

Keywords: X-ray fluorescence, phase contrast imaging, synchrotron, trace elements

\footnotetext{
${ }^{*}$ Corresponding authors. Address: ESRF, 6 rue J.Horowitz, 38000 Grenoble, France. Tel: +33476881754 (E.Kosior), +33476882650 (P.Cloetens), Fax: +33476882785

Email addresses: ewelina.kosior@esrf.fr (E.Kosior), peter.cloetens@esrf .eu (P.Cloetens) 


\section{Introduction}

Metals present as trace elements in biological systems play an important role in the cell metabolism [1], [2]. In any organism most of the intracellular processes are regulated by metals, such as proteins containing metal cofactors which catalyze biochemical reactions. Present in enzymes, they are involved in important biosynthetic pathways including the conversion of electrochemical to chemical energy, the biosynthesis of DNA, and an array of important metabolites [3]. The number of studies on trace elements is growing and nowadays their role in human health both in their natural occurance and via therapeutic drugs is recognized as crucial. Compounds containing metals are used for the diagnoses and treatment of diseases such as Alzheimer, Parkinson, cancer (treatment with cisplatin-based drugs in chemotherapy [4]) and to develop nanocomposites for gene therapy. Quantitative study of the distribution of trace elements at the sub-cellular level provides important information for the study of the functions and pathways of metalloproteins and therapeutic approaches, especially in connection with the local chemical state of the element. To answer fundamental questions on the role of metal ions in these systems requires combination of different approaches across the multidisciplinary fields of biology, chemistry and physics.

The relevant information about trace elements in biological specimens increases with the sampling resolution. To probe the sub-cellular complexity of metal ion homeostasis mechanisms, one requires sub-micrometer resolution together with a sub-femtogram absolute detection limits due to the decreasing quantity of sample probed. X-ray fluorescence (XRF) analysis with a nanoprobe is the most direct and sensitive method to quantify the distribution of metals and other elements at the sub-cellular level [5], [6]. It provides high sensitivity for transition metals and other relevant trace elements together with the capability of penetrating and mapping whole cells.

Most of the details of cells are undetectable in hard X-ray microscopy due to the weak absorption contrast between structures with similar transparency. However the various organelles show wide variation in refractive index, that is, the tendency of the materials to bend light, providing an opportunity to distinguish them. The technique exploiting the refractive index (its real part) is phase contrast imaging. Methods based on phase contrast provide complementary information about cells by mapping the electron density. Information about the electron density combined with the average density of the sample allows to calculate its thickness, value which can be compared with alternative techniques (Scanning Transmission Ion Microscopy, Atomic Force Microscopy). We use such comparison for validating the method described in this article. In this work we limit our investigations to the $2 \mathrm{D}$ regime. Therefore the quantitative values are averaged over the sample thickness. The local concentrations would be accessible by combining the proposed method with 3D tomography techniques. Without going to the full 3D investigation the proposed method allows to avoid misinterpretations of X-ray fluorescence maps, which would result from the inability to distinguish if a change in fluorescence signal is due to a change in concentration or a change in sample thickness.

Our method (combining XRF with phase contrast imaging) yields the projected concentrations at the sub-cellular level after the sample preparation and thus it is sensitive to it. This represents possible future approach - to study at the sub-cellular level the influence of different sample preparation techniques on the cell integrity. Although, not discussed in the present paper, another interesting possibility would be to investigate the radiation damages of the sample and to correct for any possible mass loss due to irradiation.

\section{Methods}

\subsection{Sample preparation}

Certified particles size standard - polystyrene spheres (Duke Scientific Corp, Palo Alto, CA) and PC12 cells were analysed. Polystyrene spheres were of four different diameters ( $400 \mathrm{~nm}, 1 \mu \mathrm{m}, 5 \mu \mathrm{m}, 10 \mu \mathrm{m})$. The commercial solution was diluted 5 to 10 times, deposited on a silicon nitride $\left(\mathrm{Si}_{3} \mathrm{~N}_{4}\right)$ membrane, spin coated and air dried at room temperature.

PC12 cells, a clonal catecholaminergic cell line derived from rat pheochromocytoma [7] were cultured in RPMI 1640 medium supplemented with $10 \%$ (v/v) heat-inactivated horse serum, $5 \%$ (v/v) fetal bovine serum, penicillin $(25$ $\mu \mathrm{g} / \mathrm{mL})$ and streptomycin $(25 \mu \mathrm{g} / \mathrm{mL})$. Cultures were maintained according to standard protocols at $37^{\circ} \mathrm{C}$ in a $95 \%$ humidified incubator with 5\% $\mathrm{CO}_{2}$ as already described [8]. The cells were cultured directly on gelatin gel coated thin polycarbonate foil ( $2 \mu \mathrm{m}$ thick). These targets were subsequently fastly cryofixed in liquid nitrogen, chilled isopentane $\left(-160^{\circ} \mathrm{C}\right)$ and further lyophilized. PC12 cells synthesize and store dopamine in neurovesicles. In the presence of nerve growth factor (NGF), these cells develop an extensive network of neuronal-like projections, that is accepted as a model 
of catecholaminergic neurons and often used as an in vitro model for Parkinson's disease. The cells labelled B,C in this work, were exposed to $300 \mu \mathrm{M}$ of $\mathrm{Mn}$ and cell A was exposed to $300 \mu \mathrm{M}$ of $\mathrm{Mn}$ and brefeldin A, a molecule known to cause the apparent collapse of the Golgi stacks [9].

\subsection{X-ray fluorescence}

$\mathrm{X}$-ray fluorescence is a phenomenon in which the material exposed to X-rays emits radiation, which has an energy characteristic of the atoms present. It allows studying the chemical composition of the sample. X-ray fluorescence analysis was performed at the nano-imaging station ID22NI (Fig.1), using the intrinsic monochromaticity of the undulators of about $\delta \lambda / \lambda \simeq 0.01$. The experimental station is located at a distance of $63 \mathrm{~m}$ from the undulator source and at $37 \mathrm{~m}$ from the high power slits used as secondary source in the horizontal direction ( $25 \mu \mathrm{m}$ slit opening).The synchrotron radiation is focused by an X-ray optical device consisting of two elliptically shaped mirrors acting in two orthogonal planes using the so-called Kirkpatrick-Baez geometry [10]. The mirrors are coated with a graded multi-layer. No other monochromator is used in the setup, resulting in a very high and unique X-ray flux (up to $10^{12}$ photons/s) at energies between 15 and $29 \mathrm{keV}$. In this work, the energy of the pink photon beam was set to 17.5 $\mathrm{keV}$ for all experiments. The focused beam was characterized by translation of a gold stripe of a nano-fabricated test pattern (Xradia, USA) recording both the transmitted intensity by a diode and the $A u-L_{\alpha}$ fluorescence emission line by a silicon drift detector (vortex-EX, SII NanoTechnology Inc., USA). The X-ray spot size was measured to be $76 \mathrm{~nm}$ horizontally by $84 \mathrm{~nm}$ vertically (FWHM). The samples were positioned in the focal plane of the KB system and translated by piezo-stages in the directions perpendicular to the beam. The X-ray fluorescence spectra of several samples were collected by the silicon drift energy dispersive detector positioned in the horizontal plane at $75^{\circ}$ of the incoming X-ray beam. Based on the X-ray fluorescence energy spectrum, the areal mass $\left(\mathrm{A}_{\text {mass }}\right)$ of the element can be calculated through a fitting procedure. The samples being scanned were deposited on $3 \times 3 \mathrm{~mm}^{2}$ membranes made of $500 \mathrm{~nm}$ thick silicon nitride $\left(\mathrm{Si}_{3} \mathrm{~N}_{4}\right)$ (Silson, Blisworth, U.K.). A step-size of $150 \mathrm{~nm}$ was used and the dwell time varied from 150 to $250 \mathrm{~ms}$.

\subsection{Phase contrast imaging}

When X-rays pass through an object, two scenarios, according to the refractive index $n$, are considered

$$
n=1-\delta+i \beta
$$

The $\mathrm{x}$-rays can be absorbed, which effect is determined by imaginary part of the refractive index, $\beta$. Image contrast is thus entirely due to absorption differences (emphasis is put on the corpuscular nature of photons) and the amplitude of the radiation changes.

The X-rays can also be retarded in the object. This phenomenon is used in phase contrast imaging, where the effects due to the decrement of the real part of the refractive index, $\delta$, are exploited. A difference in the $\delta$ value between an object and the background results in a phase shift between the wave transmitted through and outside the object. Due to this phase shift an interference pattern appears upon propagation. Emphasis is thus put on the wave nature of $\mathrm{x}$-rays. The phase shift induced by the object can be described as

$$
\phi(x, y)=-(2 \pi / \lambda) \int \delta(x, y, z) d z
$$

where $z$ is the propagation direction and $\lambda$ the wavelength.

Far from the absorption edges and considering the fact that the elemental content is dominated by the cellular architecture, comprised of light elements $(\mathrm{C}, \mathrm{N}, \mathrm{H}, \mathrm{O})$ in biological systems, the real part of the refractive index is approximately proportional to the density of the object, $\rho$.

$$
\delta \approx 1.36 \times 10^{-6} \rho\left[\mathrm{g} / \mathrm{cm}^{3}\right] \lambda^{2}[\AA]
$$

thus the equation 2 can be simplified

$$
\phi=\alpha\langle\rho\rangle T
$$

where $\alpha$ is a known constant, $\langle\rho\rangle$ is the mean density and $T$ is the thickness of the object, $\langle\rho\rangle T$ represents also the areal mass density of the cellular ultrastructure, denoted hereafter the projected mass. 
Combining the information from the X-ray fluorescence map (the areal elemental mass) with the projected mass measured by the phase contrast one can calculate the projected elemental concentration:

$$
C_{i}=A_{\text {mass }}^{i} /\langle\rho\rangle T
$$

where $C_{i}$ is a projected concentration of the element $i$ averaged over the sample thickness.

The same experimental setup as described in 2.2 was used for the magnified phase contrast imaging but the sample was put downstream of the focus in the divergent beam (see Fig.1). Projection microscopy, which uses partial coherence of the incident beam is a simple way of phase contrast imaging through propagation [11], [15]. Samples were put at different distances $(29 \mathrm{~mm}, 30 \mathrm{~mm}, 34 \mathrm{~mm}, 44 \mathrm{~mm}$ ) downstream of the X-ray focus resulting in magnified Fresnel patterns collected by a highly sensitive charge-coupled device (CCD) camera. The need of multiple distances is related to the phase retrieval procedure [16]. The pixel size varied depending on the distance focus-sample, being set to 53 $\mathrm{nm}$ for the first distance (while the pixel size of the detector was $0.96 \mu \mathrm{m}$ ).

\subsection{Atomic Force Microscopy (AFM) measurement}

Atomic Force Microscopy allows imaging the surface morphology at nanoscale resolution and measuring the force at nano-Newton scale. AFM can investigate thin and thick film coatings, synthetic and biological membranes, metals, polymers, semiconductors and study locally the electric, magnetic or mechanical properties of the sample [17]. Thanks to its versatility, AFM became a tool of choice in biophysics [18]. Our measurements were performed in Tapping mode under ambient conditions on an Asylum MFP-3D AFM instrument equipped with acoustic box. In this MFP-3D AFM the planar scan relies on an $(\mathrm{X}, \mathrm{Y})$ scanner in closed loop while an independent piezoelectric element drives the $\mathrm{Z}$ displacement of the probe according to the open feedback loop control. A standard Tapping probe was used with a measured resonant frequency of $325 \mathrm{kHz}$ and a cantilever stiffness of about $45 \mathrm{~N} / \mathrm{m}$ (average value provided by the manufacturer data sheet). The AFM topographic images were recorded with a numerical resolution of 256 x 256 pixels. The tapping mode allows high-resolution topographic imaging of surfaces that are easily damaged or not hold tightly to their substrate. The Tapping mode is implemented in ambient air by exciting the cantilever assembly near the cantilevers resonant frequency using a dedicated piezoelectric crystal. Thanks to this mechanical excitation, the cantilever oscillates with amplitude of typically few tens of nanometers; this amplitude is modulated when the tip comes into contact with the surface. The AFM measurement was performed on polystyrene spheres of 5 $\mu \mathrm{m}$ diameter at the Surface Science Laboratory of the ESRF.

\subsection{Scanning Transmission Ion Microscopy (STIM)}

Scanning Transmission Ion Microscopy is based on measurement of the energy loss of accelerated ions after passing through the specimen. This slowing down of the charged particles occurs during inelastic collisions with electronic shells and is proportional to the stopping power of the sample and describes its atomic density. The experiment was performed at AIFIRA ion beam facility on nanobeam line [19]. Charged particles delivered by a 4 MV CockroftWalton electrostatic accelerator are focused onto the sample surface by an electromagnetic lens down to a beam spot size of $200 \mathrm{~nm}$. The beam is scanned over the surface in order to measure local ion energy loss using a passivated implanted planar silicon (PIPS) detector placed on the beam axis very close behind the sample. This geometry results in increased detection efficiency, which enables drastic lowering of the beam current down to a few hundreds of particles per second. Therefore, the sample does not suffer any mass loss during ion interaction and one can access the projected mass of the sample [20].

\subsection{Data analysis}

\subsubsection{X-ray fluorescence}

For X-ray fluorescence analysis we used PyMCA, a free available software, which was developed at the European Synchrotron Radiation Facility [21]. The program allows interactive as well as batch processing of large data sets and is well suited for X-ray fluorescence imaging. Quantification of the elemental areal masses is based on a fitting procedure of the recorded fluorescence spectra. The algorithms employed are described in detail elsewhere [21]. 


\begin{tabular}{|c|c|c|c|}
\hline Element & Standard value $(\mu g / g)$ & Experimental value $(\mu g / g)$ & Difference $(\%)$ \\
\hline Potassium & $9940 \pm 20$ & $7200 \pm 9$ & -27.6 \\
Copper & $160 \pm 8$ & $169 \pm 0.2$ & 5.6 \\
Iron & $184 \pm 15$ & $166 \pm 0.3$ & -9.8 \\
Zinc & $127 \pm 16$ & $128 \pm 0.2$ & 0.8 \\
\hline
\end{tabular}

Table 1: Certified Values of Constituent Elements of the NIST SRM 1577B bovine liver together with the experimental values and their statistical uncertainties and the percentage of difference

\subsubsection{Calibration of the fluorescence data}

The Standard Reference Material (SRM) of bovine liver was used to calibrate the fluorescence spectra. This material is intended primarily for use as a control material and in evaluating analytical methods for the determination of major, minor and trace elements in animal tissue and other biological matrices.

The certified values for the constituent elements and the experimental values are given in Tab.1. Certified values are based on results obtained by definitive methods of known accuracy, or alternatively, from results obtained by two or more independent analytical methods. The grand mean was computed using the weighting scheme of Paule and Mandel [22].

The bovine liver sample was scanned through the focal plane and the spectrum of emitted fluorescence was recorded. The elemental content is calculated assuming the fundamental parameters (flux, detector characteristics) to be known except for the distance between the focus and the detector. This distance is not precisely known but it's evaluated by comparison of the calculated and known elemental content in the bovine liver sample.

Three elements were chosen as representative $(\mathrm{Fe}, \mathrm{Cu}, \mathrm{Zn})$ and three distances close to the physical one. Using PyMCA the content of the chosen elements were obtained and used in the formula:

$$
Y(x)=\sum_{i}\left(c_{i}^{s t}-c_{i}^{e x p}(x)\right)^{2}, i=\mathrm{Fe}, \mathrm{Cu}, \mathrm{Zn}
$$

where $x$ is the distance between the focus and the detector, $c_{i}^{s t}$ is a content of the element $i$ from the standard values, $c_{i}^{e x p}(x)$ is a content of the element $i$ measured in the experiment. The cost function $Y(x)$ was fitted with a second order polynomial and the value of $x$ giving the minimum was taken to be true focus to detector distance.

\subsubsection{Phase imaging}

To analyze phase contrast data we used free, available ImageJ software and the phase retrieval code developed at the ESRF and implemented under GNU Octave ([23], [24]). The results obtained by using the phase retrieval algorithm allow to calculate the thickness of a certain area (as described in 2.3). This value can be compared with the alternative methods (AFM, STIM).

\section{Results}

\subsection{Reference samples}

Certified polystyrene spheres of well known diameter were used for validation of the phase retrieval algorithm. Samples were put at four distances as explained in 2.3. The phase retrieval algorithm provides relative phase maps (Fig.2). We draw profiles across the spheres to get information on the projected phase that can be used to calculate the thickness of the sample (as described in 2.3). By introducing the density of the certified polystyrene spheres that is $1.05 \mathrm{~g} / \mathrm{cm}^{3}$ we obtained the results shown in Tab.2. It provides the nominal values of the spheres diameter (certified values), the experimental values and the relative differences. It can be observed that the experimental values are systematically underestimated and that the relative difference is bigger for the smaller spheres. The sample that consists of $5 \mu \mathrm{m}$ polystyrene spheres was also imaged by AFM and STIM, as they represent alternative methods for the thickness measurement. The results are presented in Fig.3 with associated profiles through one sphere. The same figure shows also a comparison between the different experimental results (phase, AFM, STIM) and the theoretical profile. STIM quantification of projected mass was performed using Paparamborde software [20]. Projected mass calculation is made by considering the slowing down of incoming particles through the bead and associated stopping 


\begin{tabular}{c|c|c|c} 
diameters & nominal $(\mu \mathrm{m})$ & experimental $(\mu \mathrm{m})$ & difference $(\%)$ \\
\hline $0.4 \mu \mathrm{m}$ & $0.4 \pm 0.004$ & $0.357 \pm 0.021$ & -10.75 \\
$1 \mu \mathrm{m}$ & $0.994 \pm 0.021$ & $0.95 \pm 0.06$ & -4.43 \\
$5 \mu \mathrm{m}$ & $4.987 \pm 0.04$ & $4.91 \pm 0.42$ & -1.54 \\
$10 \mu \mathrm{m}$ & $10.03 \pm 0.05$ & $9.77 \pm 1.07$ & -2.59
\end{tabular}

Table 2: The nominal and experimental values obtained on the polystyrene spheres together with their statistical uncertainties and the percentage of difference

power variation along the ion path. The ion path is shematically divided into elementary sub-layers with constant energy loss where stopping power and corresponding mass can be calculated. Total projected mass is then calculated giving weight to every sub-layer according to the total transmitted energy spectra. In the case of the polystyrene sphere calculation, the $\mathrm{Si}_{3} \mathrm{~N}_{4}$ contribution to the total energy loss was measured directly on transmitted map and used as a fixed parameter for sphere projected mass calculation. Accuracy in STIM mass measurement is driven by the nature of sample and background and remains here under $16 \%$ for the less favourable case $\left(5 \mu \mathrm{m}\right.$ sphere on $\mathrm{Si}_{3} \mathrm{~N}_{4}$ layer).

\subsection{Element mapping in PC12 cells}

PC12 cells were imaged by synchrotron X-ray fluorescence and X-ray phase contrast imaging. After scanning the sample (as described in 2.2) and fitting the emitted spectra, the intracellular distribution of the accessible chemical elements can be determined at a spatial resolution corresponding to the beam size $(100 \mathrm{~nm})$. By applying the phase retrieval algorithm, the relative phase maps were generated. The potassium $(\mathrm{K})$ and zinc $(\mathrm{Zn}) \mathrm{X}$-ray fluorescence map and phase contrast images after phase retrieval reconstruction acquired on typical NGF-differentiated PC12 cells are presented in Fig.4. Fig. 6 shows images of the same PC12 cell obtained using the STIM method and both X-ray fluorescence and X-ray phase contrast imaging. For every cell we calculated the minimal and maximal areal mass of the selected elements; potassium which is known to have a rather uniform distribution within the cell and zinc. The results are collected in Table 3. Using data from the reconstructed phase maps, similarly to what has been done for

\begin{tabular}{|c|c|c|c|c|}
\hline \multirow{2}{*}{ Cells } & \multicolumn{2}{|c|}{$\mathrm{K}$} & \multicolumn{2}{c|}{$\mathrm{Zn}$} \\
\cline { 2 - 5 } & $\min \left(\mathrm{g} / \mathrm{cm}^{2}\right)$ & $\max \left(\mathrm{g} / \mathrm{cm}^{2}\right)$ & $\min \left(\mathrm{g} / \mathrm{cm}^{2}\right)$ & $\max \left(\mathrm{g} / \mathrm{cm}^{2}\right)$ \\
\hline cell A & $15.0 \cdot 10^{-9}$ & $1.9 \cdot 10^{-6}$ & 0 & $16.4 \cdot 10^{-9}$ \\
cell B & 0 & $7.3 \cdot 10^{-6}$ & 0 & $67.1 \cdot 10^{-9}$ \\
cell C & $6.5 \cdot 10^{-8}$ & $2.8 \cdot 10^{-5}$ & 0 & $85.0 \cdot 10^{-9}$ \\
\hline
\end{tabular}

Table 3: Values of areal mass of $\mathrm{K}$ and $\mathrm{Zn}$ in PC12 cells.

the reference samples, we can calculate the thickness of the PC12 cells analysed. The profiles along selected lines (shown in Fig. 4, 6) were used for calculation. Applying an average density of the dry cells of $1.218 \mathrm{~g} / \mathrm{cm}^{3}$ [25],[26] we calculate the maximum thickness of the cell A (along the selected profile) to be $2.87 \mu \mathrm{m}$. For the cell B we selected 2 profiles, one going through the cell (profile 2) and one going through a thin neuritic process between 2 cells (profile 3). The thickness of these areas are respectively: $6.17 \mu \mathrm{m}$ and $1.28 \mu \mathrm{m}$. For the $\mathrm{Zn}$ fluorescence map of the cell B we selected also region of significant content of $\mathrm{Zn}$, visible on the Fig. 4 and the corresponding region of high projected mass. We used this region to calculate the local areal mass of $\mathrm{Zn}\left(51.16 \cdot 10^{-9} \mathrm{~g} / \mathrm{cm}^{2}\right)$ and the thickness of this part $(7.59 \mu \mathrm{m})$. These numbers will be used to calculate the absolute projected concentration of $\mathrm{Zn}$ in this region of the cell. Also for the cell C we selected 2 profiles, one going through the cell (profile 4) and one going through the lower part of the cell (profile 5). The thickness of these areas are respectively: $4.77 \mu \mathrm{m}$ and $0.82 \mu \mathrm{m}$.

On the STIM map of the cell $\mathrm{C}$ we selected 3 areas that were used for areal mass calculation. The area $\mathrm{X}$ was selected in one of the thinnest parts of the cell (similarly like profile 5 on the corresponding phase map) and it gave the result of $88 \mu \mathrm{g} / \mathrm{cm}^{2}$ while the result obtained by using the phase map gives $100 \mu \mathrm{g} / \mathrm{cm}^{2}$. The area Y goes through the nucleus of the cell (similarly like profile 4 on the corresponding phase map) and it yields $524 \mu \mathrm{g} / \mathrm{cm}^{2}$. The similar area selected 
on the phase map gives a result of $581 \mu \mathrm{g} / \mathrm{cm}^{2}$. As a curiosity we also calculate the areal mass in area $\mathrm{Z}$, which goes through the cytoplasm, the result is $114 \mu \mathrm{g} / \mathrm{cm}^{2}$ (with $150 \mu \mathrm{g} / \mathrm{cm}^{2}$ calculated from the phase map).

After combining the information from X-ray fluorescence maps and phase contrast maps it is possible to calculate the absolute projected concentration for elements $\mathrm{Zn}$ and $\mathrm{K}$ in the cells (as described in the section 2.3). For these calculations and for easier comparison we used the maximal value of the areal mass and the thickness of the cell (along the selected profiles), although full quantitative maps can be derived combining both types of images (see Fig.5). The results are presented in Table 4. For the region of the cell B (significant content of $\mathrm{Zn}$ ) mentioned above we calculate

\begin{tabular}{c|c|c} 
& $\mathrm{K}(\%)$ & $\mathrm{Zn}(\mu g / g)$ \\
\hline cell A & 0.54 & 46.9 \\
cell B & 0.97 & 89.3 \\
cell C & 4.82 & 146.3
\end{tabular}

Table 4: Projected concentration of $\mathrm{K}$ and $\mathrm{Zn}$ in PC12 cells obtained by dividing the maximal value of areal mass by the thickness of certain area.

the absolute projected concentration of $\mathrm{Zn}$ at the level of $55.3 \mu \mathrm{g} / \mathrm{g}$.

In the Fig. 5 we present the quantitative map of the projected concentration of $\mathrm{Zn}$ in cell B. By using the X-ray fluorescence map of $\mathrm{Zn}$ and the phase contrast map of the cell B (Fig.4) it is possible to access the projected concentration by dividing both maps pixel by pixel. Before the division we used cross correlation techniques to align the images and we applied small distortion corrections. It should be noted that it is not necessary to calculate the thickness of the sample in order to determine the projected concentrations of a given element as this information is accessible by dividing $\mathrm{X}$-ray fluorescence and phase contrast images pixel by pixel (see equations 4, 5). By doing the above calculations and comparing the results obtained by our method in terms of sample thickness and areal mass with an alternative method such as STIM, we validate our method.

\section{Discussion}

An important application of X-ray fluorescence microscopy is the determination of the distribution of the chemical elements at the sub-cellular level. Indeed, XRF analysis is a multi-element analytical technique that allows the simultaneous quantification of almost all elements in an unknown sample. The case of cells or thin sections of tissue is ideal in the way that corrections for absorption and enhancement effects are not necessary resulting in linearity between fluorescence intensity and the projected mass of a given element. However, the mapping of true element concentration at the sub-cellular level is a difficult task. Locally, the composition and the density or the thickness of different sub-cellular compartments can vary. Indeed, cell organelles are a mixture of structures with different size, weight and shape. The region of the cell nucleus is a few microns thick while some parts of the cytoplasm can be only $100 \mathrm{~nm}$ thick. It has been reported using combined STIM and AFM method that within a same cell specific densities vary between 1.06 to 1.86 [20]. This should be taken into account for true elemental quantification at subcellular level. To address this problem, X-ray fluorescence sub-cellular mapping and propagation-based X-ray phase contrast imaging for mass normalization were combined, for the first time, to provide high-resolution, truly quantitative, elemental distribution maps in cells. Phase-contrast imaging with a partially coherent beam produces Fresnel interference fringes, which make it possible to image samples with small variations in mass density. These variations would be undetectable in absorption contrast.

Alternatively scanning differential phase contrast imaging has been performed by measuring the deviation of the beam by the sample with a position sensitive detector [11]. The authors have demonstrated a very good sensitivity of this approach using scanning transmission X-ray microscopy on 5 microns polystyrene spheres. Although, they did not use it yet for mass normalization for X-ray fluorescence quantitative analysis, the results obtained were done at low energy $(2.5 \mathrm{keV})$ that limits the range of elements accessible. Recently the studies on Zernike Phase Contrast have shown the possibility of obtaining the projected phase in scanning mode [12]. Compton to Rayleigh scattering analysis has been used to obtain more accurate quantification in $2 \mathrm{D}$ [13] or 3D [14] measurements. There is limitation to this approach, so far the matrix mean atomic number is estimated from calculated Compton/Rayleigh intensity ratios as a function of the average atomic number in various samples which not necessarily match the sample matrix studied. In addition the thickness needs to be determined from thickness calibration curves on the basis of the total Compton 
scatter intensity. On the overall these average values based on Compton signal seem not suited to provide absolute quantification at the sub-cellular level. The projection X-ray microscopy method used in our paper is optimized for relatively high energies $(17 \mathrm{keV}-29 \mathrm{keV})$ that match well with efficient excitation of the X-ray fluorescence. As a fullfield CCD based approach our method is more dose efficient, provides a larger field of view and is much faster than scanning methods to obtain the phase information. For comparison, the phase images are acquired with a few seconds of exposure time and contain 4 million pixels ( $1 \mu \mathrm{s} / \mathrm{pixel})$, whereas the fluorescence scanning data requires about 6 hours of exposure time for 100000 pixels $(250 \mathrm{~ms} /$ pixel). However the phase data is not recorded simultaneously with the XRF data as it is in [12]. Therefore to normalize the fluorescence data the alignment of the two different data sets is required. The higher energy of excitation for X-ray fluorescence allows not only a better sample penetration but allows also to reach almost all element with $Z>13$ with the advantage to work with $\mathrm{K}$ emission lines for higher fluorescence yield and better energy resolution.

Ion beam techniques have shown clear assets to obtain true elemental quantification by combining particle induced $\mathrm{X}$-ray emission (PIXE) with Rutherford backscattering and/or STIM. The sensitivity and spatial resolution are much poorer than synchrotron-based XRF microscopy. Recently, efforts have been done towards the quantification of trace elments on cell cultures [8]. STIM allows to map with sub-micrometer resolution the variation in mass densities within single cells with calculated thicknesses in good agreement with AFM measurements [20]. In this work, first a comparison between reference techniques (AFM, STIM) was made on polymer microspheres to mimic, in size and composition, a typical biological specimen such as a cell. The X-ray phase imaging after the application of a phase retrieval reconstruction algorithm provides a map of the projection of the mass density of the sample that was converted to thickness knowing the polystyrene density. The calculated thickness profile for 5 microns spheres was found to be slightly underestimated using X-ray phase imaging. This can be explained by the shape of the background of the profile extracted from the X-ray phase signal and that is not corrected for. The varying background is the direct consequence of the sensitivity of differential phase contrast methods for low frequency noise. This problem can be solved by improving the quality and stability of the beamline optics, resulting in a better correction for inhomogeneities of the incoming X-ray beam. The STIM data matches more closely the theoretical profile of the sphere. Here, microspheres were deposited on $500 \mathrm{~nm}$ thick silicon nitride membranes. The 2 layers, i.e the sphere and the silicon nitride membrane were separated using STIM calculation modified in accordance. There is not much discrepancy between STIM - phase - and theoretical profile as shown in Fig 3. The AFM topographic image provides two complementary measurements through the profile: a $4 \mu \mathrm{m}$ in height and $5.1 \mu \mathrm{m}$ for lateral dimension (measured at the half height of the microsphere, i.e $2 \mu \mathrm{m}$ where the tip-object convolution effect can be neglected). These differences in height and lateral dimensions could be due to the absence of non-linearity correction of the piezoelectric element in $\mathrm{Z}$ direction while the planar scanner is corrected thanks to the closed feedback loop. Indeed, the AFM instrument is dedicated to accurate height measurements at sub-micronic scale where the non-linearity behavior can be neglected. For this reason, in our measurements, the lateral one is the more reliable. Based on this assumption, the lateral dimension matches with the diameter specification of the sphere data sheet.

The thickness measurements using X-ray phase images were in good agreement (within 5\% except for the smallest spheres) when compared to the certified values for the sphere. We attribute the increased relative error for the smallest spheres to a spatial resolution effect: in the phase retrieval step we didn't take into account the finite point spread function of the detector. The quantitative measurements on single $400 \mathrm{~nm}$ spheres are encouraging for subcellular applications. Indeed, this typically matches the size of some organelles (lysosome, mitochondria) or some thin cellular processes (neurites, filopodia). This is well exemplified when PC12 cells were analyzed. These cells were differentiated into neuronal-like cells which, expands neuritic processes. The X-ray phase reconstructed images provide useful structural details giving evidence for sub-cellular compartments of various densities and a cell nucleus that can be well delineated. Further work will be needed for better identification of the structural details obtained in the phase images by coupling to fluorescence microscopy and organelle staining. We observed inside some PC12 cells denser regions, micrometric in size. The X-ray fluorescence signal of zinc was found much higher in these regions. While the zinc distribution can be first misinterpreted, the association of X-ray phase images (for mass normalization) to $\mathrm{X}$-ray fluorescence quantification results in a projected zinc concentration that remains rather similar to the one in other parts of the cell (see Fig. 5). The potassium signal is found homogeneous as expected [8]. This indicates that the cell integrity was preserved during the sample preparation. The areal mass obtained on PC12 cells through STIM and X-ray phase measurements are in good agreement both for a thick part of the cells like the nucleus and for thinner parts like neurites. These direct comparisons on the same cellular regions support the use of X-ray phase imaging 
for mass normalization in X-ray fluorescence quantification. The same cells were imaged by STIM, then by X-ray phase imaging followed by X-ray fluorescence measurements. Despite short acquisition times (150-250 ms) and low attenuation at high X-ray energies, the high X-ray photon flux delivered by the nanoprobe results in non-negligible radiation damage. It is suggested that some mass loss occurs and our approach could be used to better characterize these effects. X-ray fluorescence microscopy is a unique tool for the quantification of trace elements at the sub-cellular level and it can be applied to a wide variety of biological studies. On the overall this work is encouraging the use of $\mathrm{X}$-ray phase imaging to improve the elemental quantification in 2D and 3D X-ray fluorescence imaging.

\section{Acknowledgements}

We would like to thank the European Synchrotron Radiation Facility for providing the beamtime. This work was supported in part by the ANR Program PIRIBIO (ANR-09-PIRI-0029-01).

\section{References}

[1] Meares, C., Wensel, T., 1984. Metal chelates as probes of biological systems. Acc. Chem. Res. 17 (6), $202-209$.

[2] Bush, AI., Curtain, CC., 2008. Twenty years of metallo-neurobiology: where to now? Eur. Biophys. J 37 (3), $241-5$.

[3] Sundberg, RJ., Martin RB, 1974. Interactions of histidine and other imidazole derivatives with transition metal ions in chemical and biological systems. Chem. Rev. 74 (4), 471-517

[4] Lippard, S. J., Berg, J. M., 1994. Principles of Bioinorganic Chemistry. University Science Books: Mill Valley, CA.

[5] Bohic, S., Simionovici, A., Snigirev, A., Ortega, R., Devès, G., et.al., 2001. Synchrotron hard x-ray microprobe: fluorescence imaging of single cells. Appl. Phys. Lett. 78 (22), 3544 - 3546.

[6] Ortega, R., Bresson, C., Fraysse, A., Sandre, C., Devès, G., et.al., 2009. Cobalt distribution in keratinocyte cells indicates nuclear and perinuclear accumulation and interaction with magnesium and zinc homeostasis. Toxicology Lett. 188, 26-32.

[7] Greene, L. A. and Tischler, 1976. A. S. Proc. natn. Acad. Sci. U.S.A. 73, 24242428.

[8] Carmona, A., Devès, G., Ortega, R., 2008. Quantitative micro-analysis of metal ions in subcellular compartments of cultured dopaminergic cells by combination of three ion beam techniques. Anal Bioanal Chem 390: 1585-94.

[9] Carmona, A., Devès, G., Cloetens, P., Bohic, S., Ortega, R., et.al., 2010. Manganese accumulates within Golgi apparatus in dopaminergic cells as revealed by synchrotron X-Ray fluorescence nano-imaging. ACS Chemical Neurosciences. 1, $194-203$.

[10] Hignette, O., Cloetens, P., Rostaing, G., Bernard, P., Morawe, C., 2005. Efficient sub 100nm focusing of hard x rays. Rev. Sci. Instrum. 76.

[11] de Jonge, M., Hornberger, B., Holzner, C., Paterson, D., Jacobsen, C., et.al., 2008. Quantitative phase imaging with a scanning transmission X-ray microscope. Phys. Rev. Lett. 100, 163902.

[12] Holzner, C., Feser, M., Vogt, S., Hornberger, B., Jacobsen, C., et.al., 2010. Zernike phase contrast in scanning microscopy with X-rays. Nature Physics 6,883-887.

[13] Vincze, L., Janssens, K., Adams, F., Rivers, M.L., Jones, K.W., 1995. A General Monte-Carlo simulation of ED-XRF spectrometers: Part II, Polarized Monochromatic Radiation, homogeneous samples. Spectrochimica Acta B, 50B, 127-147.

[14] Golosio, B., Simionovici, A., Somogyi, A., Lemelle, L., Chukalina, M., et.al., 2003. Internal elemental microanalysis combining X-ray fluorescence, Compton and transmission tomography. J. of Appl. Phys. 94, 145-156.

[15] Di Fabrizio, E., Cojoc, D., Cabrini, S., Kaulich, B., Susini, J., et.al., 2003. Diffractive optical elements for differential interference contrast X-ray microscopy. Opt. Express 11, 2278-2288.

[16] Mokso, R., Cloetens, P., Maire, E., Ludwig, W., Buffiere, J.Y., 2007. Nanoscale zoom tomography with hard x rays using Kirkpatrick-Baez optics. Appl. Phys. lett. 90, 144104.

[17] Marchi, F., Dianoux, R., Smilde, H.J.H., Mur, P., Comin, F., et.al., 2008. Characterisation of trapped electric charge carriers behavior at nanometer scale by electrostatic force microscopy. Journal of Electrostatics, pp538547.

[18] Alessandrini, A., Facci, P., 2005. AFM: a versatile tool in biophysics. Meas. Sci. Technol. 16, R65-R92.

[19] Barberet, Ph., Incertia, S., Andersson, F., Delalee, F., Serani, L., et.al., 2009. Technical description of the CENBG nanobeam line. Nucl. Instrum. and Methods in Phys. Res. Section B: Beam Interactions with Materials and Atoms. 267, 12-13.

[20] Devès, G., Cohen-Bouhacina, T., Ortega, R., 2004. Scanning transmission ion microscopy mass measurements for quantitative trace elements analysis within biological samples and validation using atomic force microscopy thickness measurements. Spectrochim. Acta B. 59, 17331738 .

[21] Sole, V., Papillon, E., Cotte, M., Walter, P., Susini, J., 2007. A multiplatform code for the analysis of energy dispersive X-ray fluorescence spectra. Spectrochim. Acta Part B At. Spectrosc. 62, 63-68.

[22] NBS Journal of Research 87, pp 377-385

[23] Cloetens, P., Ludwig, W., Baruchel, J., V.Dyck, J., V.Landuyt, J., et.al., 1999. Holotomography: Quantitative phase tomography with micrometer resolution using hard synchrotron radiation x ray s. Appl. Phys. Lett. 75, 2912-2914.

[24] Langer, M., Cloetens, P., Guigay, JP., Peyrin, F., 2008. Quantitative comparison of direct phase retrieval algorithms in in-line phase tomography. Med. Phys. 35(10), 4556-66.

[25] Snyder, W. S., Cook, M. J., Nasset, E. S., Karhausen, L. R., Howells, G. P., et.al., 1975. Report of the Task Group on Reference Men. International Commission on radiological protection No. 23. Pergamon Press Oxford.

[26] Carmona, A., Devès, G., Ortega, R., 2008. Quantitative micro-analysis of metal ions in subcellular compartments of cultured dopaminergic cells by combination of three ion beam techniques. Analytical and Bioanalytical Chemistry 390(6), $1585-1594$. 




Figure 1: Scheme of the experimental setup of the nano-imaging station ID22NI 

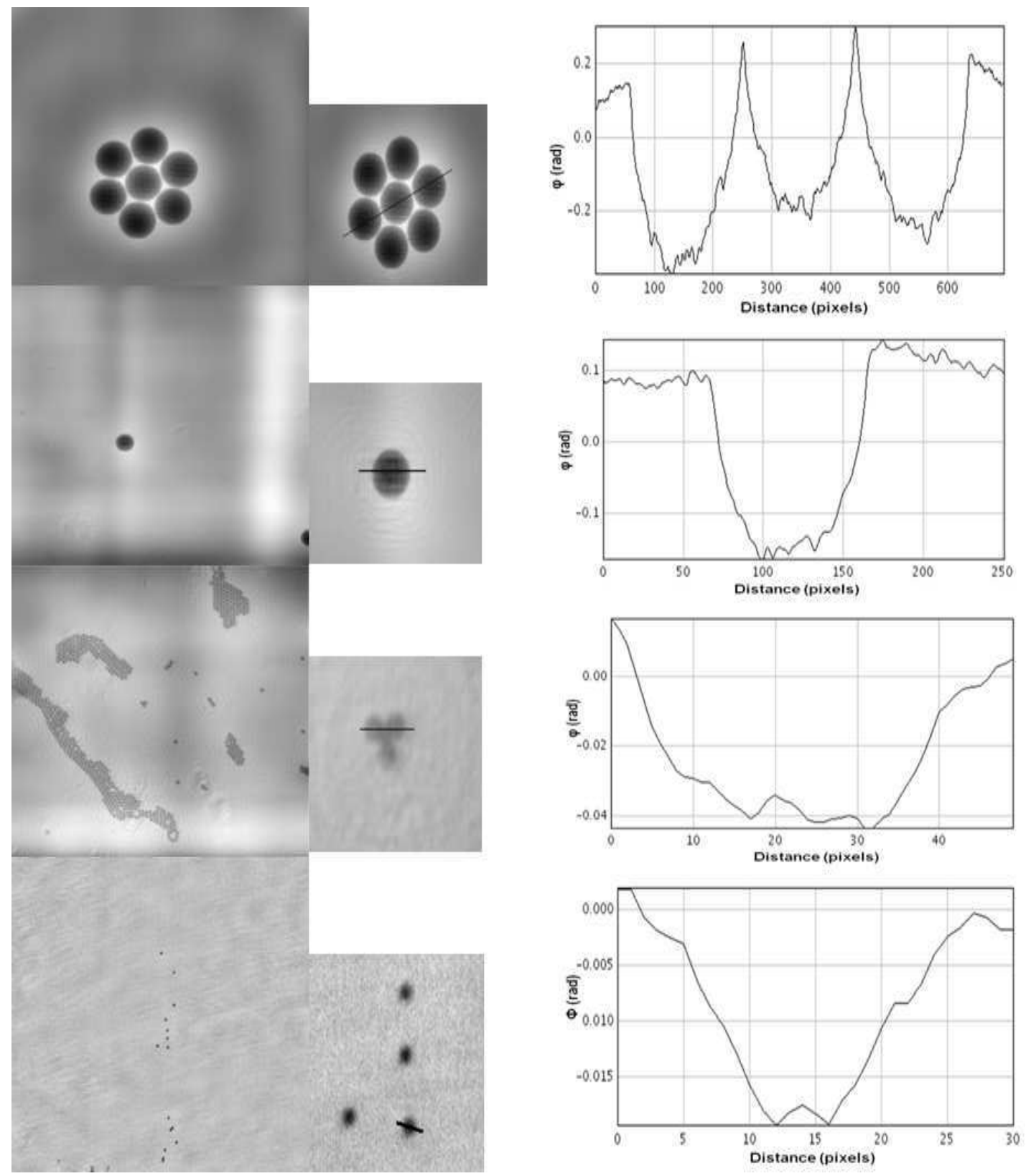

Figure 2: Relative phase maps generated after applying phase retrieval procedure to the certified polystyrene spheres of following diameters: 10.03 $\pm 0.05 \mu \mathrm{m}, 4.987 \pm 0.040 \mu \mathrm{m}, 0.994 \pm 0.021 \mu \mathrm{m}, 400 \pm 4 \mathrm{~nm}$, together with selected profiles. 

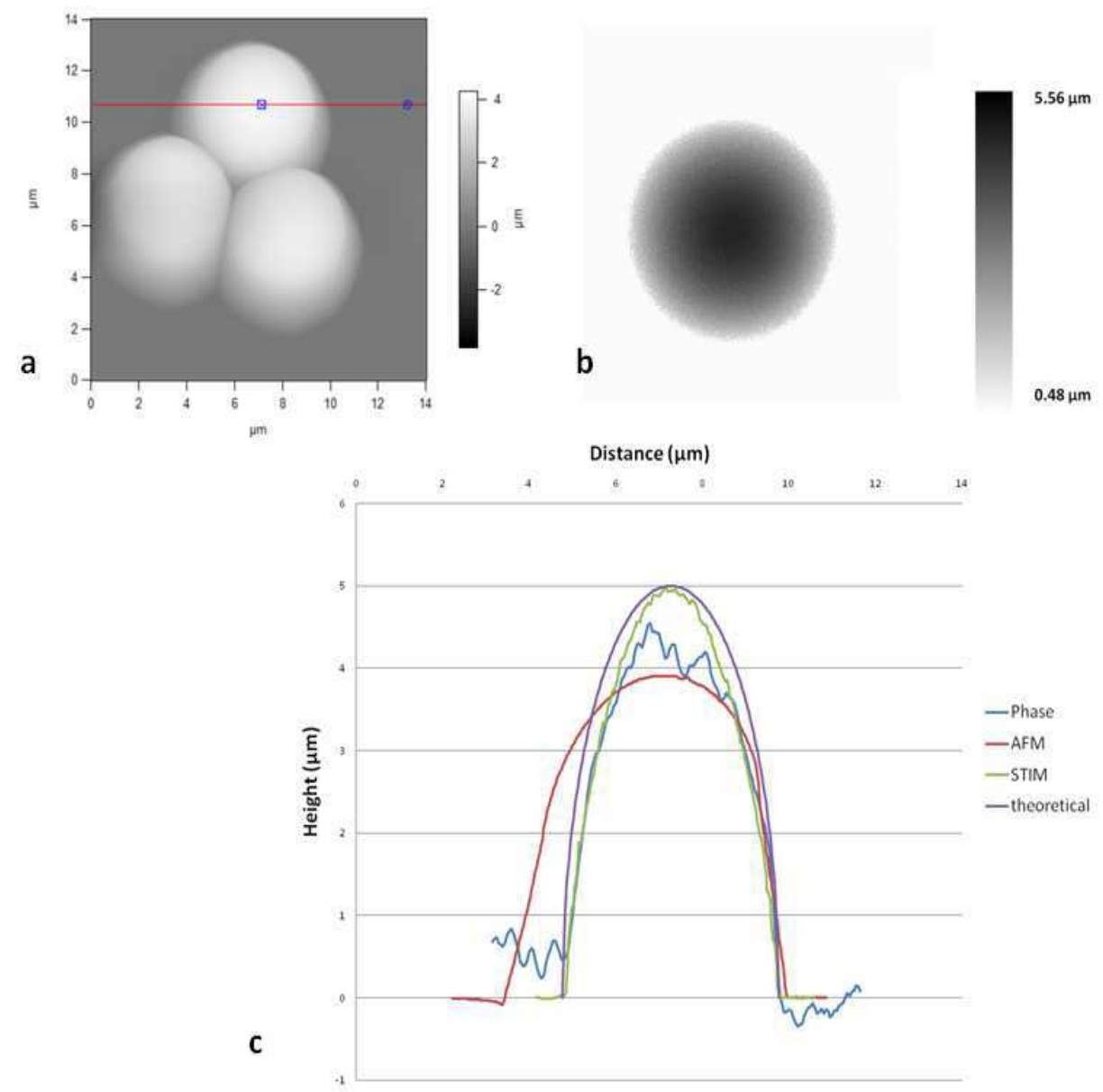

Figure 3: Comparison between different imaging techniques of the $5 \mu \mathrm{m}$ spheres. a. the AFM image of the $5 \mu m$ spheres, b. STIM image of the 5 $\mu \mathrm{m}$ sphere, c. comparison between the experimental profiles (phase, AFM and STIM) and the theoretical prediction. 


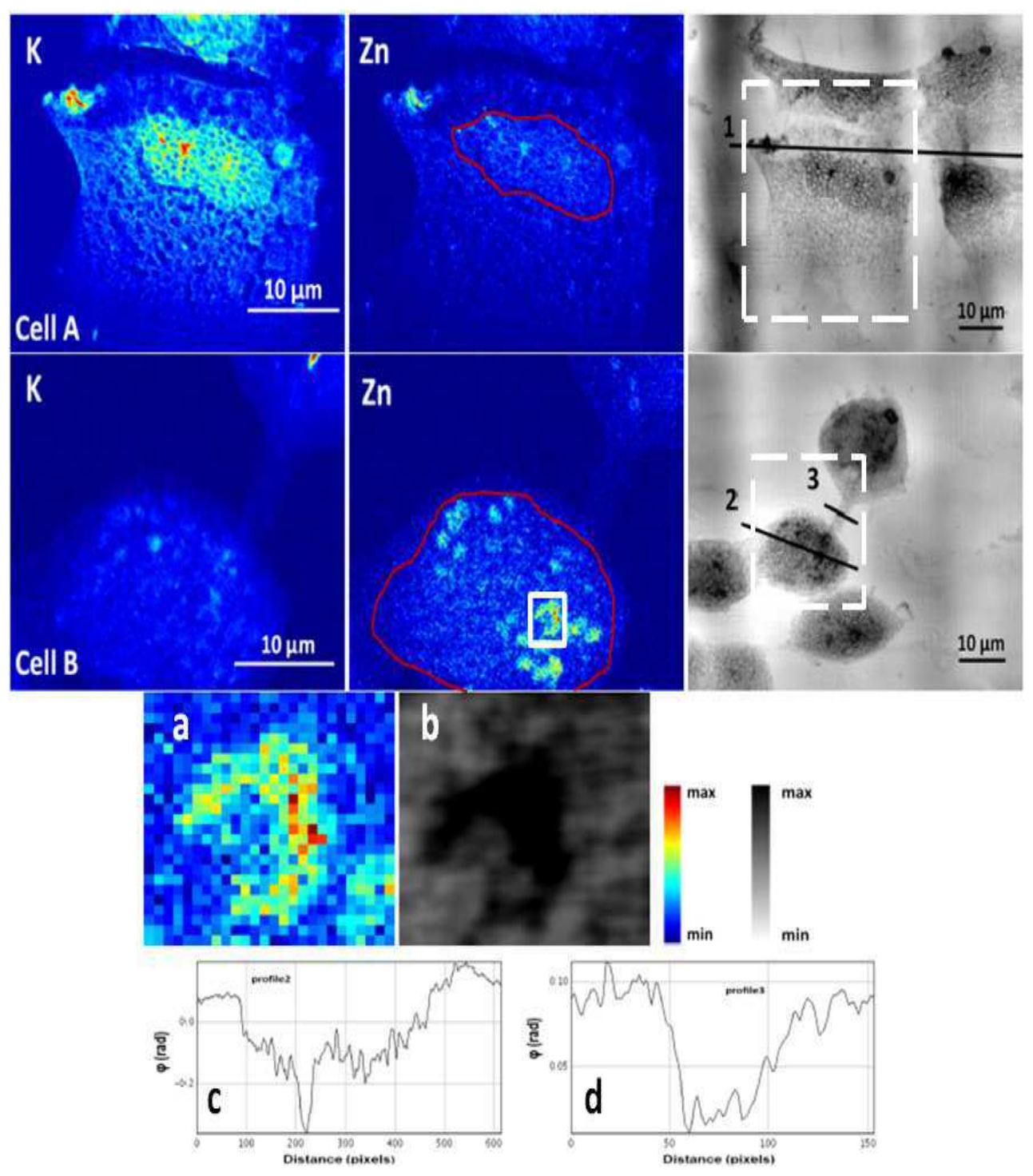

Figure 4: X-ray fluorescence (color) and phase contrast (black-white) maps of PC12 cells A and B. Dashed, white squares on phase contrast maps indicate the corresponding region on fluorescence map. The cell nuclei are highlighted in red. White square on $\mathrm{Zn}$ fluorescence map of the cell B was selected due to significant content of Zn (fluorescence map - a) and thickness (phase map -b); c. Profile 2, through the cell B; d. Profile 3, through the neuritic process. The colors depicting elemental content in each map are scaled to the maximum value for that map. The scanning size of the cell A was 307 x 308 pixels with dwell time 250 ms while for the cell B 194 x 176 pixels with dwell time of $150 \mathrm{~ms}$ 


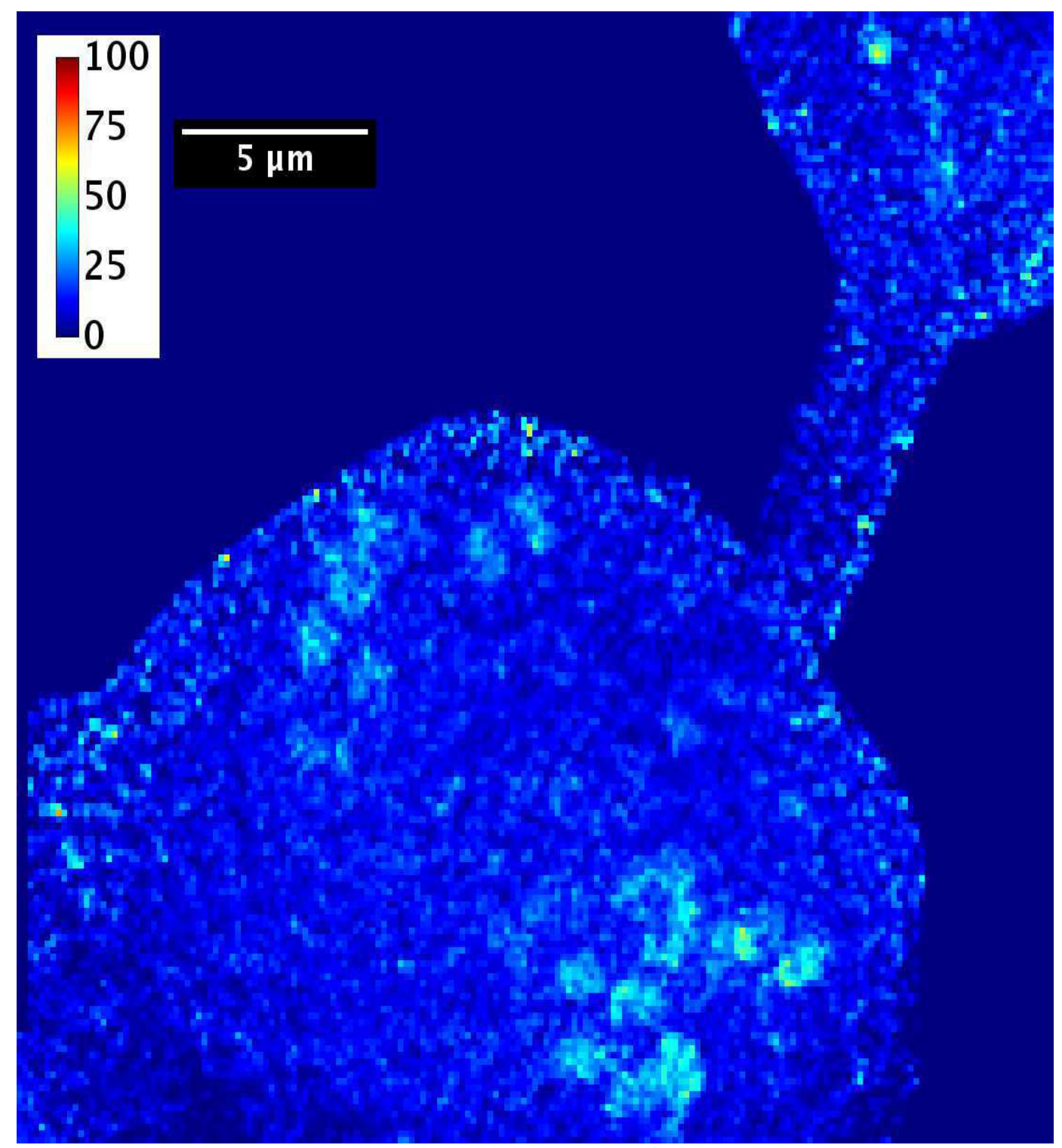

Figure 5: Fully quantitative map showing the projected concentation of $\mathrm{Zn}$ in the cell $\mathrm{B}$. The calibration bar is in ppm. The map was created by combining the X-ray fluorescence map of $\mathrm{Zn}$ and the phase map of the cell $\mathrm{B}$ by dividing both images pixel by pixel. 


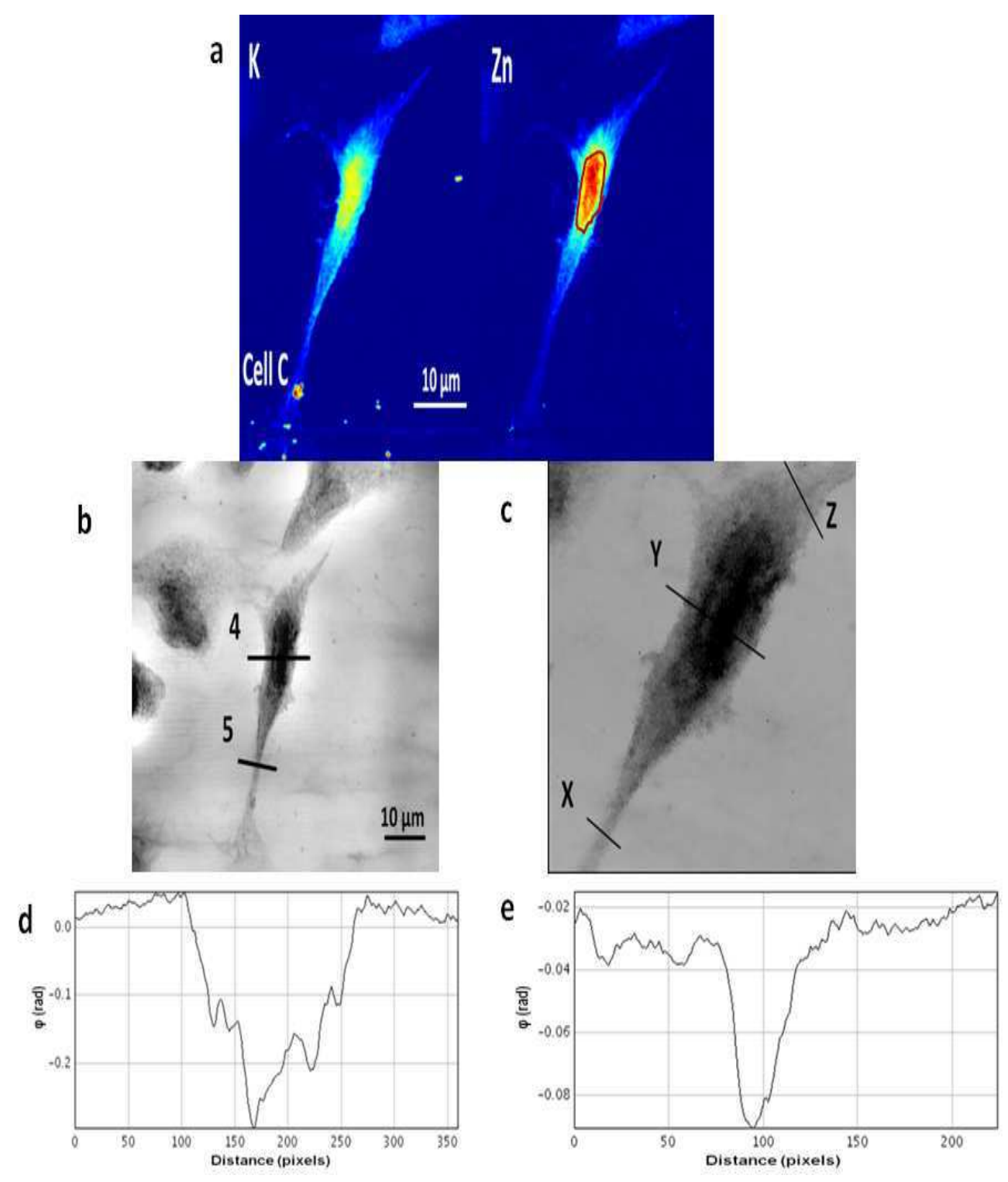

Figure 6: X-ray fluorescence (a), phase contrast (b) and STIM (c) map of the cell C. The cell nucleus is highlighted in red. On the phase map there are 2 areas selected for the thickness calculation; d. Profile 4 through the cell C, e. Profile 5 through the lower part of the cell. On the STIM map there are 3 selected areas X, Y, Z, which were chosen to calculate the areal mass. The colors depicting elemental content in each map are scaled to the maximum value for that map. The scanning size was 225 x 411 pixels and dwell time $200 \mathrm{~ms}$. 